\title{
A simple method for validation and verification of pipettes mounted on automated liquid handlers
}

Stangegaard, Michael; Hansen, Anders Johannes; Frøslev, Tobias Guldberg; Morling, Niels

Publication date:

2009

Document version

Publisher's PDF, also known as Version of record

Citation for published version (APA):

Stangegaard, M., Hansen, A. J., Frøslev, T. G., \& Morling, N. (2009). A simple method for validation and verification of pipettes mounted on automated liquid handlers. Poster session presented at LabAutomation2009, Palm Springs 2009, United States. 


\section{A simple method for validation and verification of pipettes mounted on} automated liquid handlers

Stangegaard M, Hansen AJ, Frøslev TG, Morling N

Section of Forensic Genetics, Department of Forensic Medicine, Faculty of Health Sciences, University of Copenhagen, 11 Frederik V's Vej, DK-2100 Copenhagen, Denmark

\section{INTRODUCTION}

Poster TP58
LabAutomation2009
Palm Springs, CA, USA

Automated liquid handlers (ALHs) are increasingly used to improve throughput, pipetting accuracy and prevent occupational injuries to the technical staff due to intensive manual pipetting ${ }^{1}$. Standardized procedures for calibration of standard piston operated pipettes exist and many laboratories calibrate their pipettes with defined intervals ${ }^{2}$. However, less standardized is the routine validation and verification of ALHs, though suggestions to how it should be performed exist ${ }^{3}$. Commercial solutions have also been introduced ${ }^{4}$.

\section{MATERIAL AND METHODS}

A 7-step serial dilution of Orange $G$ was prepared manually in quadruplicates in a flat bottom 96-well microtiter plate (BD Falcon) by means of calibrated pipettes (column 9-12). This was used as a standard row. Each pipette of the

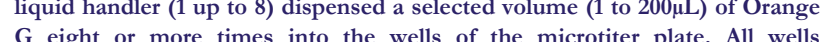
$\mathrm{G}$ eight or more times into the wells of the microtiter plate. All wells contained a total of $200 \mu \mathrm{L}$ liquid. The optical density (OD) was read at 490 $\mathrm{nm}$, and the of volume and OD of a known set of Orange G dilutions. Finally, the perce
inaccuracy (\%d) and the imprecision (\%CV) of each pipette was calculated.

Figure 1. Plate layout. A standard row is included on each plate.

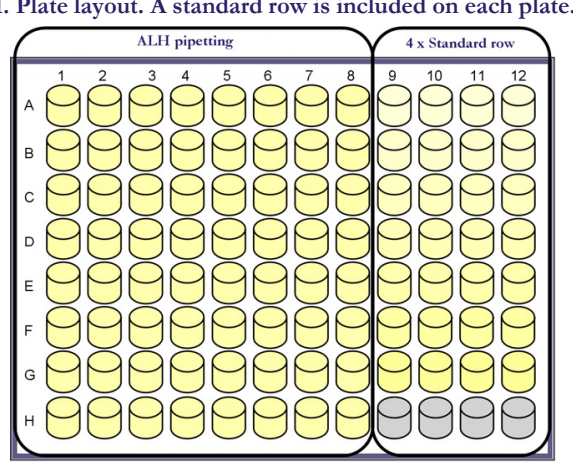

Figure 2. Volume verification using manual pipetting and a conventional pipette set to 2.0 $\mathrm{L}$. Pipette number refers to different runs.

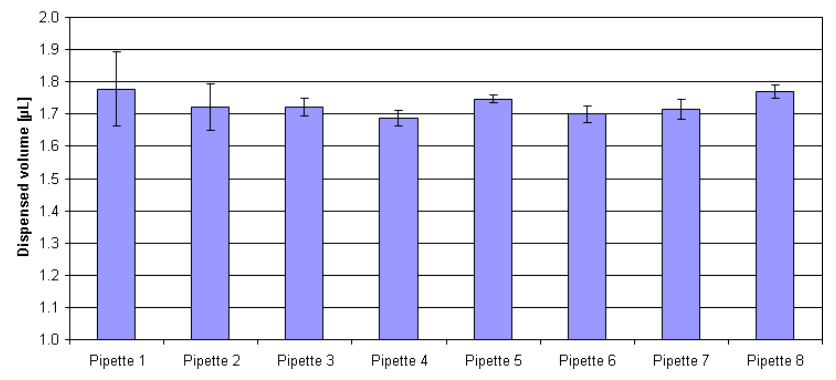

\section{PROCESS FLOW}

The process is composed of few and simple steps. The same stock solution may be used for verification of multiple ALHs. A calibrated ALH may be used to prepare the standard row. Initial calibration was performed with manually pipetted standard rows.

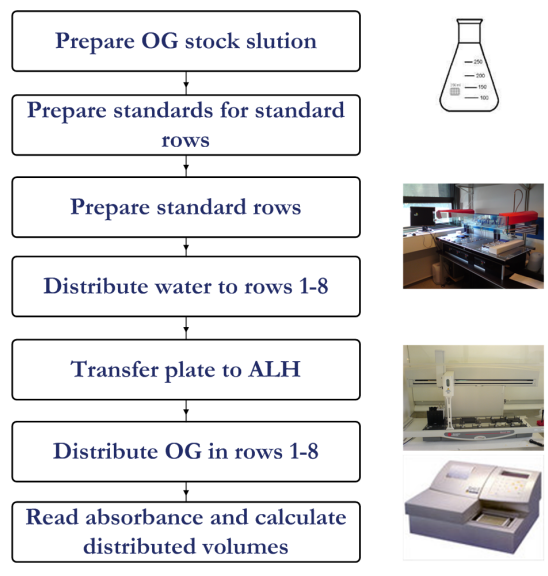

\section{RESULTS}

Using pre-defined acceptance criteria, each pipette on each ALH was then either approved or rejected. Rejected pipettes were either repaired or the volume deviation was compensated for by applying a calibration curve in the liquid handler software. We have implemented the method on a Sias Xantus, a MWGt TheONYX, four Tecan Freedom EVO 150, a Biomek NX Span-8 and four Biomek 3000 robots.

Figure 3. Two different volume verification runs both using $50 \mu \mathrm{L}$ as target volume on a 4 channel fixed tip MWG TheONYX robot.

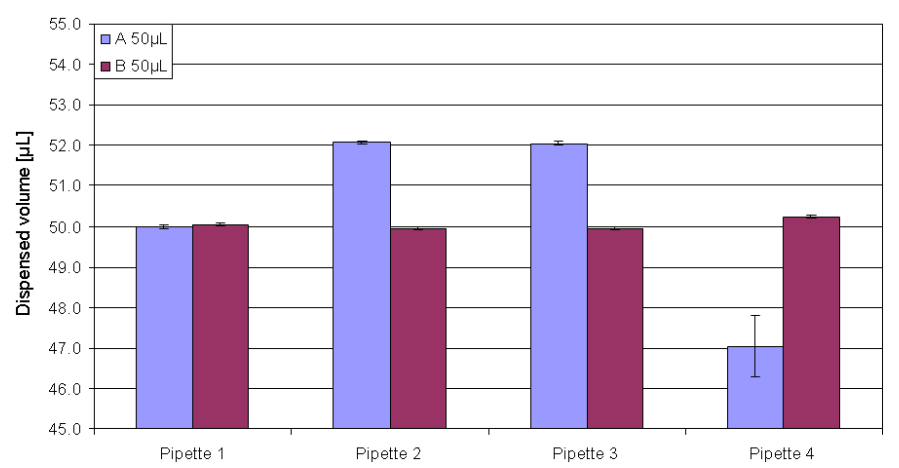
Figure 4: Two different volume verification runs both using $5.0 \mu \mathrm{L}$ as target volume on an eight chan fhowed unpipetting.

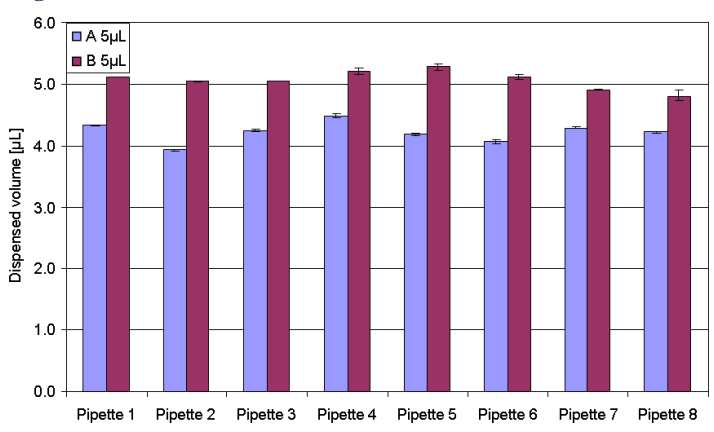

Figure 5. Three different volume verification runs all using $1.0 \mu \mathrm{L}$ as target volume on a Biomek NX span-8 using disposable tips. Run A and Run B show either too little or too large volume dispensed. Run C shows acceptable pipetting.

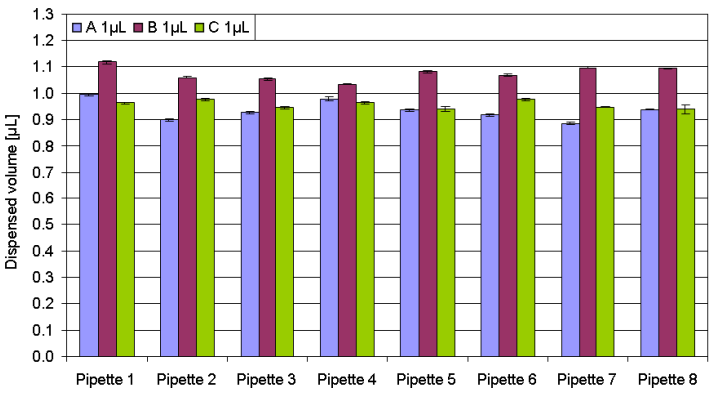

\section{CONCLUSIONS}

We have set up and implemented a simple solution for the continuous verification of pipettes mounted on automated liquid handlers as necessary fo accredited work under the international laboratory standard $15 \mathrm{O}$ 17025. The method is cheap, simple and easy to use for aqueous solutions, but it requires a spectrophotometer that can read microtiter plates. The method can be used with both disposable tips, fixed tips as well as manual pipetting.

\section{REFERENCES}

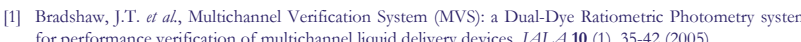
[2] Batista, E. et al, Volume calibration of 1000 pll micropipettes. Inter-maboratory comparison. Accreditation and

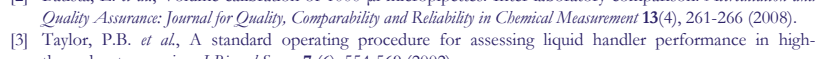

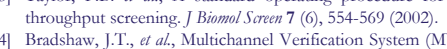
Bradshaw, J.T, et all, Multichannel Verification System (MVS): a Dual-Dye Ratiometric Photometry system
for performance verification of multichannel liquid delivery 